\title{
Le sfide di «un universo più fluido» nell'opera di Giorgio Fontana
}

Parole chiave: immigrazione, legge e giustizia, spazio urbano multietnico, cronotopo, vita liquida, romanzo sociologico.

DOI: $10.4312 /$ ars.10.2.108-120

\section{Prospettive}

Nelle opere con cui si è profilato all'attenzione della critica internazionale Giorgio Fontana va a rodere quella posizione scettica che è stata dominante negli ultimi decenni e che continua a improntare largamente i nostri anni. Di fronte a una società composta in gran parte da individui arresi, attraversati da un profondo senso d'impotenza sulle sorti del mondo e portati quindi ad accettare una vita di compromessi, questo scrittore fa appello a un soggetto capace di mettersi in gioco fino in fondo, e ciò anche in un tempo privo di certezze.

In più sensi i suoi romanzi Per legge superiore (del 2011) e Morte di un uomo felice (del 2014) s'incentrano su cambiamenti di prospettiva, ma innanzitutto circa il modo d'intendere la coscienza civile e di praticare la giustizia. Per evidenziarlo si potrebbe iniziare da un episodio finale del primo dei due lavori, che è anche quello su cui ci si soffermerà nel seguito. Il protagonista fa notare alla sua interlocutrice un'iscrizione posta sulla parete posteriore del Palazzo di giustizia a Milano, Fiat iustitia ne pereat mundus (Sia fatta giustizia perché non muoia il mondo) e precisa che quella frase è un rimaneggiamento di epoca fascista. In precedenza l'iscrizione prevedeva un et al posto del ne e quella sostituzione ne mutò in maniera rilevante la semantica. La frase originaria Fiat iustitia et pereat mundus (Sia fatta giustizia e il mondo muoia pure) guardava alla giustizia senza esaltarne il fine salvifico, ma accentuando semmai che il perseguirla non lascia spazio a compromessi: la si compie, costi quel che costi.

Una revisione di prospettiva viene vissuta in prima persona anche dal protagonista del romanzo, figura di sostituto procuratore in attesa di un'ultima promozione che appaghi una certa sua ambizione professionale. Dopo essere stato coinvolto da una giovane giornalista socialmente impegnata a riconsiderare un caso giuridico - e a farlo da unottica per lui nuova, non più basata sulla mera logica dei fatti cui si era 
sempre affidato («[...] soltanto le cause interessano, solo la fisica del male e non l'etica») $)^{1}-$, l'uomo si trova a vivere una fase di ripensamenti. Ne danno atto non solo una flessione della sua metodicità o la nuova distanza con cui ascolta un rampante giurista sostenere a un convegno che «l'etica e l'accertamento non avevano nulla da condividere», ${ }^{2}$ ma anche il frequente bisogno di ritoccare sul suo computer (fino poi a cancellarlo) un testo autobiografico intitolato Testamento dove aveva iniziato a fissare le proprie posizioni etiche e professionali. ${ }^{3} \mathrm{E}$ così, fra titubanze se restare o no fedele ai suoi principi di condotta, questo maturo magistrato, compiaciuto di essere stato sempre un uomo retto, arriva poi al punto di mettere in questione la sua fede in una giustizia esercitata nel rispetto della legge quale ultima salvaguardia dellordine («perché l'alternativa è il caos $[\ldots] »)^{4}$ e sembra essere pronto ormai a praticarla esponendosi «in prima persona $\aleph^{5}$ ben sapendo che ciò comporterà sconvolgimenti nella sua vita. Vista dal punto di vista della teoria del personaggio, tale evoluzione avrebbe quasi potuto incontrare (con un ipotetico salto indietro di una settantina d'anni) il favore del Debenedetti di Personaggi e destino, critico preoccupato delle sorti del personaggio novecentesco in quanto figura di soggetto incapace a mettersi pienamente in gioco. Solo che nella riflessione debenedettiana la nekuia ${ }^{6}$ era un salto in abisso d'altro formato.

Sul faticoso cambiamento d'ottica, maturato nel personaggio di Fontana in un dialogo conflittuale con la giovane giornalista tacciata d'idealismo, ruota l'intera vicenda del romanzo. Quanto a sé, l'uomo si dichiara persona disincantata «[...] il disincanto è l'unica teoria in grado di spiegare gli esseri umani, proprio perché non fornisce consolazioni.» ${ }^{7}$ Se ha un credo, è di tipo laico, come viene specificando in un punto del suo Testamento:

Credo che ci sia una luce. Una fiamma che è la Giustizia e che dobbiamo proteggere con le mani dal vento. È un'immagine banale, ma non sono un letterato, e dopotutto ritengo che per queste cose la banalità sia una virtù, un mezzo per andare più lontano[...]. Certo, Giustizia e legge possono differire in maniera significativa, ma in questi tempi bui l'interrogazione sulla prima non può che ridursi al rispetto verso la seconda $[\ldots] .^{8}$

1 Fontana, 2011, 107.

2 Ivi, 196.

3 Acuto il rilievo che dà a tale motivo, nella sua recensione al romanzo, Giorgio Vasta (2012).

4 Fontana, 2011, 35.

5 Ivi, 228.

6 Debenedetti, 1994, 125.

7 Fontana, 2011, 66.

8 Ivi, 72-73. 
L'immagine della fiamma da tenere riparata perché continui ad ardere verrebbe evidentemente al magistrato dalla sua confidenza con l'opera di de La Tour; in più occasioni ripete infatti di essere colpito dall'elemento di fragilità con cui la luce è resa nelle tele del pittore. Quando non sarà più del tutto convinto che la giustizia poggi interamente sulla legge (sfuggendo talvolta la legge alla complessità della realtà), egli inizierà a vedere anche le scene di quei quadri con occhi diversi: della riproduzione di una tela che tiene in ufficio, il San Giuseppe, lo colpirà piuttosto la strana confusione fra la parte di dipinto rappresentante la realtà e quella rappresentante il sogno. ${ }^{9}$

La riflessione sul rapporto fra legge e giustizia, sviluppata dunque anche attraverso il ricorso all'ecfrasi, ha nellopera una portata generale, ma prende avvio da un caso giuridico specifico in cui è coinvolto un immigrato. La storia pretende che l'accusato di una sparatoria, in cui rimane paralizzata una ragazza, sia un giovane muratore tunisino stranamente pronto ad ammettere la sua colpevolezza, sebbene gli indizi raccolti per via informale dalla giornalista free lancer lo mostrino innocente. Il sostituto procuratore, cui spetterebbe solo di valutare la correttezza del processo, viene quindi trascinato in un'indagine parallela. E tale motivo, che sulle prime può farci sospettare di avere fra le mani un racconto poliziesco, permette all'autore di veicolare il discorso su una problematica sociale e di lì su questioni etiche e identitarie. Il testo apre così delle considerazioni sulla società europea alle prese con un'immigrazione massiccia che sta trasformando l'identità degli spazi metropolitani, dei codici di vita e delle forme di convivenza. Lattenzione di Fontana si concentra nella fattispecie sui cambiamenti di Milano. Sulle stesse questioni verteva già un precedente lavoro dello scrittore, Babele 56, diverso tuttavia per genere e accostabile semmai all'inchiesta documentaria o meglio a quella scrittura ibrida che, partendo da documenti autentici, non si vieta un'elaborazione letteraria.

Nella narrativa italiana dell'ultimo ventennio tra gli esempi maggiormente comparabili a Per legge superiore viene in mente Sostiene Pereira di Tabucchi. ${ }^{10}$ Quell'opera si reggeva in verità su un'altra poetica ed era costruita con differenti strategie narrative, ma Per legge superiore mostra degli elementi analoghi tanto nella trama (il combattuto abbandono di certe posizioni da parte del protagonista fino a un atto di coscienza civile conseguente al nuovo orientamento), quanto in precisi motivi. Anche qui è una persona giovane a lanciare l'impulso a un uomo maturo a rivedere convinzioni consolidate e anche qui il protagonista, in una fase di dubbi, sente la necessità di cercare consiglio diretto o mentale in chi rappresenta per lui un'autorità morale (un vecchio professore di diritto e uno stimato collega morto assassinato). Invece dal punto di vista poetologico la posizione di Fontana potrebbe

9 Cfr. ivi, 240.

10 Tabucchi, 1994. 
essere accostata semmai a quella di narratori contemporanei che, come rilevato dal comparatista Emiliano Ilardi, non sono più concentrati ad attivare l'attenzione del lettore sui processi narrativi, ma la convogliano su problematiche dell'attualità e recuperano così il romanzo sociologico ponendo appunto «come fonte delle loro narrazioni il conflitto tra le differenti concezioni e percezioni del mondo oggi presenti.» ${ }^{11}$ Per quanto il tipo di conflitto e di personaggi osservabili nella narrativa esaminata da Ilardi, incentrata sui fenomeni della paranoia e della devianza, siano ben diversi da quelli proposti da Fontana, potremmo considerare anche il suo testo come un romanzo sociologico a patto che tale proposta non sia troppo delimitante; Per legge superiore si lascia infatti considerare anche come unopera di carattere morale e di riflessione sull'identità. Per quanto concerne la forma, il testo propone una scrittura all'insegna della chiarezza e ha come sua peculiarità un ampio uso dell'immagine significante. Non presenta soluzioni complesse né a livello di struttura e nemmeno di scelte discorsive e linguistiche. Vi possono anzi risultare perfino ripetitive le formule prescelte per rendere il contesto spaziale ed emozionale in cui ha luogo unazione o una situazione (con l'immancabile richiamo a esperienze sensitive di luce, suono, odore). La conduzione del racconto è affidata a un narratore onnisciente in terza persona: fuori dai dialoghi e dalle immaginarie dichiarazioni autobiografiche è tale voce a restituire i dubbi e le incertezze del protagonista senza che v'intervengano soluzioni retorico-discorsive di altro tipo, come vuoti di parola o contraddizioni. E ciò solo per specificare che il disorientamento del protagonista non diventa mai, in questo caso, anche quello del lettore. Altro preme evidentemente allautore: non sottolineare i limiti o l'inaffidabilità della lingua, ma semmai valorizzarne l'uso comunicativo. Per riprendere la tesi sopra avanzata circa la presenza di cambiamenti di prospettive, si potrebbe dunque aggiungere che le scelte di scrittura abbracciate da un narratore della più recente generazione, come Fontana, marcano anche un rovesciamento di prospettive poetologiche rispetto alla generazione postmodernista dei suoi 'padri'.

\section{Spazi}

L'ipotesi da cui muove questa lettura riprende la riflessione bachtiniana sullo spazio narrativo integrandovi anche alcune tesi difese da Bertrand Westphal, diffusore dell'approccio geocritico. Si postula che il discorso condotto nel romanzo si organizzi in maniera decisiva attraverso i suoi cronotopi, ma si riconosce contemporaneamente che lo spazio, nei termini in cui se ne parla nel romanzo, è un'entità esposta al

11 Ilardi, 2005, 28. Ilardi avanza delle considerazioni pertinenti sulle svolte narrative in atto negli anni di svolta del secolo; ci pare tuttavia eccessivamente netta la distinzione da lui rilevata tra metaromanzo postmodernista e nuovo romanzo sociologico. Come se la narrativa che veicola l'attenzione del lettore sui processi narrativi si vietasse necessariamente uno sguardo sui conflitti sociali. 
cambiamento, pluristratificata e costruita anche attraverso la percezione che ne hanno i personaggi. ${ }^{12}$

Sono due luoghi urbani a costituire l'asse attorno ai quali si configura il sistema di relazioni testuali. Il primo è uno spazio chiuso, il Palazzo di giustizia di Milano; il secondo uno spazio aperto, una strada nella zona periferica della città. Costruzione imponente e austera, rivestita da lastre di marmo, il Palazzo di giustizia, si rivela a un esame ravvicinato in uno stato tutt'altro che solido: sembra non aver retto un massiccio ampliamento ai piani superiori e sta cedendo. Come un leitmotiv, la sua immagine torna costantemente nelle pagine del romanzo attraverso lo sguardo del protagonista che continua a restare colpito da come ledificio mostri i segni del tempo e la sua fragilità:

Da lontano sembravano solo imperfezioni o macchie naturali delle lastre: e invece erano chiodi, grossi chiodi a espansione in metallo: un modo per tenere saldo il marmo, visto che la malta originale stava per cedere e l'intero edificio era a rischio.

Quegli oggetti avevano qualcosa di morale, naturalmente. Il luogo della Giustizia piegato alle leggi più alte della materia $\left[. . .{ }^{13}\right.$

Anche il movimento delle persone al suo interno non appare ben regolato: «faceva parte della logica del Palazzo: una zona dove le regole erano incerte, l'opposto di ciò che doveva essere: chiodi e crepe, come sempre.» ${ }^{14}$ Quello spazio viene dunque ripetutamente collegato ai concetti di fissità e corrosione. S'insiste sugli intonaci che cadono, sulle macchie di umidità, sullodore di chiuso che vi emana. Guardando il Palazzo dal retro, il protagonista osserva che «sembrava un galeone incagliato» ${ }^{15}$ e ancora, dall'interno, constata che: «[...] sulle scale lodore di sigaretta e stantio si era fatto quasi insopportabile. Tutto sembrava ancora più statico [...].» ${ }^{16}$ Il Palazzo, per la stessa ridondanza che riceve nel testo (è menzionato già nell'incipit) nonché per il modo in cui si collega agli altri elementi testuali, parla oltre la lettera. Come lo stesso protagonista suggerisce, lo si può prendere a significante di un sistema giuridico divenuto elefantiaco e vulnerabile, ma anche (in forma di sineddoche) per quel cuore

12 Westphal, 2004, 82. Tale studio, come pure quello più ampio uscito per le Édition de Minuit (Westphal, 2007) accoglie in maniera sincretica teorie filosofiche e letterarie d'indirizzo semiotico, accomunate dal rifiuto (centrale nel postmoderno) di un logos basato sui principi di uniformità e staticità e in particolare dalla necessità di considerare anche lo spazio in termini di eterogeneità e fluidità. Il discorso sviluppatovi non distingue sufficientemente l'ambito letterario da quello sociologico e politico, ma ciò s'inscrive con tutta evidenza nel voluto approccio interdisciplinare.

13 Fontana, 2011, 13.

14 Ivi, 31, corsivo di chi scrive.

15 Ivi, 23.

16 Ivi, 30. 
regolamentato della città che, da "galeone incagliato», non arriva sempre a trovare un legame con le isole periferiche, o ancora per un'istituzione (politica o sociale) consolidata, ma impreparata a reagire ai mutamenti. Non da ultimo si lascia assumere come figura di una forma mentale.

Il giorno in cui lei gli scrisse, Doni pensò che il Palazzo aveva subito quel destino perché rifiutava lo spazio circostante. Lo combatteva, incapace di appartenere a quella come a qualsiasi altra zona della città. [...]

Era qualcosa che aveva a che fare con l'esilio. ${ }^{17}$

Laltro luogo che organizza gli elementi testuali in sistema, funzionando in collegamento con lo spazio del Palazzo, è via Padova. Si tratta di una lunga strada della zona nord, popolata da immigrati di tante nazionalità. In quell'area, «in un'altra Milano», ${ }^{18}$ dove non era ancora mai stato, il magistrato Doni si sente fuori luogo. E però a metterlo a disagio non è tanto il senso di estraneità, quanto la povertà. Anzi a un certo punto ammette che l'edificio dalla «facciata sfatta», ${ }^{19}$ dove abita la sorella dell'accusato, gli ricorda la propria infanzia, solo che ora nel cortile ci sono dei bambini dalla pelle scura. Fra costruzioni che vanno alla deriva coglie comunque della dignità. ${ }^{20}$ In un bar di fortuna, con arredi invecchiati da anni '70, avverte perfino un'atmosfera confortevole. Ma soprattutto in via Padova resta colpito da qualcosa di sensitivo, dall'assenza dellomologato, da una presenza di voci, colori, odori. Come se la vita pulsasse in altro modo rispetto alla Milano che conosceva. ${ }^{21}$

E poi quellodore. Ecco il particolare che gli era sfuggito. Milano non era una città da attraversare con i sensi, lo scenario naturale di una passeggiata: [...] Milano era insapore, inodore - era un luogo fatto di negazioni. Per quello la amava, in fondo: perché era qualsiasi cosa e niente insieme. Eppure via Padova sfuggiva a quel teorema. ${ }^{22}$

Se la descrizione di via Padova e dei suoi dintorni cede in qualche passo al topico fascino dell'esotico, quel quadro trova un contrappeso nella menzione di alcuni lati ben meno solari del luogo: il degrado edile, appunto, ma anche alcune situazioni conflittuali fra i gruppi che vi abitano, nonché lo spaccio di droga. E ciò nel senso di fondo del discorso affrontato, orientato a dare voce alla complessa identità di luoghi.

17 Ivi, 13-14

18 Ivi, 95.

19 Ivi, 85.

20 Cfr. ivi, 97.

21 Il motivo del ritrovato senso della vita nelle periferie ritornerà anche in Morte di un uomo felice nella narrazione di un altro periodo della storia italiana.

22 Fontana, 2011, 95. 
Gli spazi che fanno da asse al sistema testuale, ben lungi dal restituire solo un'immagine di grandangolo dell'attuale paesaggio urbano di una grande città italiana, nella loro funzione cronotopica entrano in relazione con il tempo e ne visualizzano i modi in cui questo si configura entro l'universo narrato. A scandire il tempo nello spazio cui più strettamente si collega l'agire del protagonista (il Palazzo di giustizia) è il lavoro. Il tempo del lavoro riempie le pause pranzo del magistrato, le soste al caffè; le sue stesse ferie, mentre la carriera gli segna il tempo della vita. Se cè memoria di un passato nella sua esistenza, così come proiezione di un futuro, queste dimensioni sono entrambe legate alla carriera. Inoltre il tempo scorre nella sua vita (di uomo metodico e privo di ogni forma di eccesso) con un ritmo regolare, da routine. Altro il delinearsi del tempo in via Padova: lì si litiga, si beve, si fuma, e certamente anche si lavora, ma il tempo della vita in quello spazio appare in ogni caso più dilatato: «Due ragazze sedute davanti alla lavanderia fumavano guardando il cielo.» ${ }^{23}$ Iniziative commerciali o legate ad attività ricreative sorgono e muoiono in quella strada con un ritmo imprevedibile.

Tramite gli spazi si delineano nel testo anche lo status e l'evoluzione del personaggio. Cedendo via, via a posizioni di maggiore flessibilità il Doni di Fontana scopre infatti un altro senso della vita assieme all'impressione di qualcosa di perso. Nel suo quotidiano lascia entrare lentamente piccole trasgressioni, inizia a compiere percorsi insoliti guardando con maggiore attenzione quanto accade attorno a lui; per la prima volta prova curiosità per i gusti musicali del fratello e ritrova perfino il tempo per un dialogo a distanza con la figlia, ricercatrice emigrata. Nel messaggio che le scrive, le confessa di cominciare a sentire più vicina una tesi da lei sostenuta tempo prima: «[...] che il mondo non funziona in bianco e nero [...] che le particelle possono essere bianche e nere insieme, e che i loro stati non sono determinabili in maniera univoca, o chiara.» ${ }^{24}$ In termini verbali fin troppo espliciti viene qui fornita una chiave di lettura del romanzo che la stessa resa dei luoghi suggerisce: il Palazzo di giustizia, con la sua fragile imponenza e via Padova, con le sue scene di esistenza precaria, condividono inaspettatamente alcuni tratti e occorre solo cambiare prospettiva per comprenderlo. Nessuno dei due spazi si esaurisce in ciò che mostra a prima vista. Analogamente, e sui vari piani del discorso (sociale, giuridico, assiologico), ogni polarità cui si accenna nel romanzo ne esce sfrangiata (legalità e illegalità, rettitudine e opportunismo, attivismo sociale e sfruttamento). ${ }^{25}$ 


\section{Fluidità}

Vedere le cose è anche un fatto di prospettiva. Nel 2008 il Fontana giornalista decide di osservare la sua «città che cambia» a partire da via Padova, la stessa via che ritorna come motivo narrativo tre anni dopo in Per legge superiore. Conduce delle interviste a immigrati stranieri che vi vivono o lavorano e le raccoglie in un testo dal titolo Babele 56, dove quella strada funge non solo da elemento strutturante il volume, ma anche da scenario per gli incontri. Otto conversazioni, immaginate come altrettante tappe del percorso dell'autobus 56, s'intercalano a nove intermezzi riflessivi di carattere sociologico-esistenziale; questi frammenti, che fungono anche da proemi alle singole conversazioni, nascono dall'osservazione di un dettaglio del luogo o di una situazione e sono espressi per lo più da un 'tu' quale 'io' che si guarda a distanza e che appella contemporaneamente lo stesso lettore. In uno di quegli intermezzi si legge: «È qui che il tuo comodo concetto di città si sgretola e perde forza.» ${ }^{26} \mathrm{E}$ una constatazione analoga a quella che sarebbe stata espressa dal protagonista di Per legge superiore a un certo punto della vicenda. In entrambi i lavori è infatti tematizzata la necessità di rivedere orientamenti e concetti che nuove situazioni hanno reso obsoleti. Anche l'identità dei luoghi cambia e non prenderne atto produce solo distanze. Alla città italiana che si conosceva, spiega l'autore introducendo Babele 56, è subentrato «un universo più fluido, caotico, arlecchino.» ${ }^{27}$ Lultimo termine che appare in questa triade segnala la varietà come elemento sostanziale a tale realtà e sembra lanciare un richiamo anche all'enorme capacità d'improvvisazione nell'arte della sopravvivenza che volens nolens è richiesta a chi la abita. Non sarà forse fuori luogo ricordare che lo stesso termine entra in relazione con un concetto di spazio mobile ed eterogeneo, «isotropico ${ }^{28}$ nello studio La géocritique: réel, fiction, espace; appare più precisamente nel paragrafo intitolato Passant à l'âme arlequine. ${ }^{29}$

Babele 56 propone uno sguardo multiplo (allogeno e endogeno) ${ }^{30}$ su una periferia urbana multietnica e sulla vita che la anima: quello dell'intervistatore, giovane di nazionalità italiana residente a Milano (ma non del tutto estraneo a quel quartiere), e quello degli intervistati, immigrati stranieri di nuova e vecchia data. I due corpi paralleli del libro - rispettivamente le otto conversazioni e i nove frammenti riflessivi - sono funzionalizzati grosso modo a restituire quelle due direzioni prospettiche

26 Fontana, $2014^{2}, 45$.

27 Ivi, 10.

28 «Lisotropie est le propre d'un espace object de mouvements, de tensions, qu'aucun ordre supérieur ne vient assujettir à une hiérarchie.» (Westphal, 2007, 65).

29 Per il termine «arlequine» Westphal fa a sua volta riferimento in quel contesto a un passo di Atlas di Michel Serres. Cfr. Westphal, 2007, 65, n. 4.

30 Westphal, 2004, 82. 
('grosso modo', poiché talvolta in una stessa conversazione le due ottiche sono compresenti). Milca, una ragazza boliviana, ricorda la sua prima occhiata dall'alto su Milano, quando la colpì la squadratura della città: «La prima impressione che ho avuto arrivando a Malpensa è stata: sembra tutto di Lego.» ${ }^{31}$ È una sorta di visione antitetica a quella che il protagonista di Per legge superiore proverà andando in via Padova. Un altro giovane intervistato, il tunisino Kais, ancora senza permesso di soggiorno, parla invece degli spazi urbani di fortuna dove bivacca: «Hai la sensazione di essere eternamente in sospeso.» ${ }^{32}$ "Essere un immigrato significa anche rimappare la città» dirà l'intervistatore in un altro contesto osservando come i luoghi vengano fortemente rifunzionalizzati in situazioni precarie di vita. ${ }^{33}$ Nell'inchiesta di Fontana è evidente soprattutto l'intenzione di evitare una prospettiva omogeneizzante. I soggetti di quelle conversazioni, rappresentano un universo sociale variegato non solo per origini etniche, ma anche per età, sesso, formazione culturale, esperienze di vita e di lavoro vissute nel Paese di arrivo. Diversificate appaiono pure le risposte degli intervistati in merito alla loro identità: per un ragazzo tunisino, musicista rap, Milano è ormai la sua città e aggiunge di non sentirsi né arabo, né tunisino, né italiano, ma "appena se stesso»; ${ }^{34}$ mentre una giovane cinese (già dall'infanzia in Italia) dichiara di sentirsi occidentale e pensa di non poter mai tornare a vivere nel suo Paese; per contro i suoi genitori si sono già-comprati una tomba in Cina. ${ }^{35}$

Lorchestra di voci che risuona in Babele 56, per riandare figuralmente a un episodio del libro, richiama la necessità di rivedere (di nuovo nel senso di un cambiamento di prospettiva) il concetto d'identità dei luoghi in una società multietnica e di pensare tale identità come qualcosa di mobile, non nettamente circoscrivibile e soprattutto non fondata solo su ciò che un luogo è stato in un certo momento della sua storia. La riflessione si allarga ovviamente anche al cambiamento della percezione della propria identità in chi li abita, i luoghi divenuti multietnici. Una simile trasformazione comporta una correzione del già saputo. Questo abbandono di posizioni che non tengono più bene non coincide con l'attitudine a eliminare di cui parla Zygmunt Bauman in riferimento alla società contemporanea. Bauman ha sostenuto nei suoi interventi, e qui potremmo ricordare nello specifico Vita liquida, che l'identità dell'individuo nella società a cavallo fra ventesimo e ventunesimo secolo (ma i tempi esigono già nuove letture) non si basa sulla conservazione bensì sulla eliminazione. «La vita liquida è una vita di consumi [...] modella secondo i canoni degli oggetti

31 Fontana, $2014^{2}, 40$.

32 Ivi, 49.

33 Ivi, 32.

34 Ivi, 85.

35 Ivi, 91. 
di consumo il giudizio e la valutazione di tutti i frammenti, animati e inanimati del mondo.» ${ }^{36}$ Una simile definizione, che trova il suo criterio orientante in un rapporto economico, quello del consumatore di fronte a merci sempre più velocemente rimpiazzate, si allarga dunque nelle riflessioni di Bauman fino a indicare uno stile di vita basato su un tipo di soddisfacimento generante subito nuova insoddisfazione - attitudine che segna anche una visione del tempo come eterno presente. Per Fontana, al contrario, il concetto di fluidità, così come affiora nei testi presi in esame, è assunto semmai dalla sfera della precarietà sociale (nel lavoro come negli altri campi dell'esistenza) e assume una diversa valenza semantica. Anche qui il concetto esce dall'ambito da cui è tratto fino a delineare una forma mentis. Si tratta in questo caso di un modo di pensare più elastico, capace di spingere il singolo a mettersi in discussione e ad abbandonare, se necessario, posizioni precedentemente ritenute per valide. Nei termini del romanzo esaminato è proprio tale forma mentis che s'insinua nel protagonista di Per legge superiore quando inizia a uscire (in doppio senso) dal suo luogo 'isola' e accoglie quanto l'anziano maestro gli ha fatto osservare e cioè che non esistono fatti, ma solo versioni. Per lui, da sempre attaccato al dato tangibile, mettersi in gioco per difendere solo una versione dei fatti (ma una versione di cui è convinto) significherà superare una frontiera mentale e intendere diversamente anche l'agire. Per lasciarlo dire a lui stesso con la sua irrinunciabile immagine-guida di un de La Tour: cessare di immaginarsi custode del fuoco e scoprire di poterne essere fiamma. ${ }^{37}$

\section{Bibliografia}

Bachtin, M., «Le forme del tempo e del cronotopo nel romanzo», in ID., Estetica e romanzo, a cura di C. Strada Janovič, Torino 1979, pp. 231-405.

Bauman, Z., Liquid life, Cambridge 2005.

Bauman, Z., Vita liquida, trad. it. M. Cupellaro, Bari 2006.

Debenedetti, G., «Personaggi e destino», ID., Saggi critici, Terza serie, Introduzione di M. Lavagetto, Venezia 1994, pp. 111-127.

Fontana, G., Babele 56. Otto fermate nella città che cambia, Milano 2014² (1. ed. 2008).

Fontana, G., Per legge superiore, Palermo 2011.

Fontana, G., Morte di un uomo felice, Palermo 2014.

Ilardi, E., Il senso della posizione: romanzo, media e metropoli da Balzac a Ballard, Roma 2005.

Tabucchi, A., Sostiene Pereira, Milano 1994.

36 Bauman, 2006, XVII.

37 Cfr. Fontana, 2011, 245. 
Vasta, G., Giudice, il dubbio sia sempre con te, Il Sole 24 Ore, inserto «Domenica», 5. febbraio 2012, http://www.ilsole24ore.com/art/cultura/2012-02-05/giudicedubbio-sempre-081416_PRN.shtml, [24. 6. 2016].

Westphal, B., Approches métodologiques de la transgression spatiale, Primerjalna književnost 27, 2004, numero speciale, pp. 75-83.

Westphal, B., La géocritique. Réel, fiction, espace, Paris 2007. 


\section{Patrizia Farinelli}

\section{Izzivi »fluidnejšega univerzuma» v delih Giorgia Fontane}

Ključne besede: imigracije, pravo in pravičnost, večetnični urbani prostor, kronotop, tekoče življenje, sociološki roman

Giorgio Fontana pogosto usmerja pogled v transformacije javnih krajev v Milanu in tako v svojih besedilih opisuje tudi spremembe kulturne identitete tega mesta. Tovrstnim spremembam se posveča v dokumentarnem delu Babele 56. Otto fermate nella città che cambia (2008), v literarni obliki pa v kratkem romanu Per legge superiore (2011). V obeh besedilih, seveda v skladu z zakonitostmi obeh žanrov, prikaže, kako množična prisotnost migrantov $\mathrm{v}$ italijanskem okolju terja ponovno ovrednotenje identitetnih koordinat teh krajev, hkrati pa pretresa tudi paradigme mišljenja, prepričanj in življenjskih slogov. Stik z množico oseb različnih etničnih pripadnosti, katerih življenje pogosto zaznamuje mobilnost in negotovost, odkriva krhkost tistega, kar se pogosto zdi trdno zasidrano v določeni kulturi: v romanu iz leta 2011 je vera $\mathrm{v}$ zakone tista, ki zahteva ponovni razmislek. 


\section{Patrizia Farinelli}

\section{The Challenges of a "more fluid universe" in the Work of Giorgio Fontana}

Keywords: immigration, law and justice, multi-ethnic urban space, chronotope, fluid life, sociological novel

In his written works, Giorgio Fontana repeatedly focuses on the transformations of the outer world of Milan in order to narrate changes to the city's cultural identity. The attention he pays to these changes is, in Babele 56. Otto fermate nella città che cambia (2008), a documentary one; in his short novel Per legge superiore (2011), it is a literary one. In both texts, and in the mode both genres require, he shows how the massive presence of migrants in Italy leads not only to reconsidering the identitymaking coordinates of locations, but also to shaking paradigms of thoughts, beliefs, and lifestyles. The contact with a crowd of people of different ethnicities whose lives are frequently characterized by mobility and precariousness enables us to discern the fragility of what is often considered unmovable into a specific culture: in his 2011 novel, it is the confidence in the law itself but also the need for a new consideration of it. 